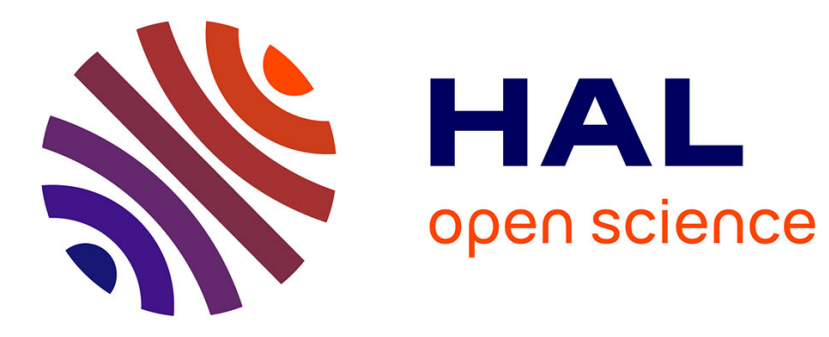

\title{
Low Complexity Joint RDO of Prediction Units Couples for HEVC Intra Coding
}

Maxime Bichon, Julien Le Tanou, Michael Ropert, Wassim Hamidouche, Luce Morin, Lu Zhang

\section{- To cite this version:}

Maxime Bichon, Julien Le Tanou, Michael Ropert, Wassim Hamidouche, Luce Morin, et al.. Low Complexity Joint RDO of Prediction Units Couples for HEVC Intra Coding. International Conference on Acoustics, Speech and Signal Processing (ICASSP), Apr 2018, Calgary, Canada. 10.1109/ICASSP.2018.8462489 . hal-01724383

\section{HAL Id: hal-01724383 \\ https://hal.science/hal-01724383}

Submitted on 6 Mar 2018

HAL is a multi-disciplinary open access archive for the deposit and dissemination of scientific research documents, whether they are published or not. The documents may come from teaching and research institutions in France or abroad, or from public or private research centers.
L'archive ouverte pluridisciplinaire HAL, est destinée au dépôt et à la diffusion de documents scientifiques de niveau recherche, publiés ou non, émanant des établissements d'enseignement et de recherche français ou étrangers, des laboratoires publics ou privés. 


\section{Low Complexity Joint Rate-Distortion Optimization of Prediction Units Couples for HEVC Intra Coding}

\author{
Maxime Bichon ${ }^{\dagger, \star}$, Julien Le Tanou $^{\dagger}$, Michael Ropert ${ }^{\dagger}$ \\ $\dagger$ Ericsson \\ France, 35136 Saint-Jacques-de-la-Lande \\ Email: firstname.lastname@ericsson.com
}

\author{
Wassim Hamidouche ${ }^{\star}$, Luce Morin ${ }^{\star}$, Lu Zhang ${ }^{\star}$ \\ * INSA de Rennes (IETR - UMR6164) \\ France, 35708 Rennes \\ Email: firstname.lastname@insa-rennes.fr
}

\begin{abstract}
HEVC is the latest block-based video compression standard, outperforming H.264/AVC by $50 \%$ bitrate savings for the same perceptual quality. An HEVC encoder provides RateDistortion optimization coding tools for block-wise compression. Because of complexity limitations, Rate-Distortion Optimization (RDO) is usually performed independently for each block, assuming coding efficiency losses to be negligible. In this paper, we propose an acceleration solution for the Intra coding scheme named Dual-JRDO, which takes advantage of Inter-Block dependencies related to both predictive coding and CABAC. The Dual$J R D O$ improves Intra coding efficiency at the expense of higher computational complexity. The acceleration of the Dual-JRDO scheme includes adaptive use of the Dual-JRDO model based on source analysis, short-listing and early decisions strategies. The proposed Fast Dual-JRDO reduces the original model complexity by $89.54 \%$, while providing tractable computation for average R-D gains of $\mathbf{- 0 . 4 5 \%}$ (up to $\mathbf{- 0 . 8 2 \%}$ ) in the HM16.12 reference software model.
\end{abstract}

Index Terms-Intra Coding, Joint Block Optimization, HEVC, Dual-JRDO

\section{INTRODUCTION}

Rate-Distortion Optimization $(R D O)$ aims to minimize the distortion $D$, subject to a target rate constraint $R \leq R_{T}$. Lagrange multiplier method is usually used to remove the constraint on $R$ [1], with $\lambda$ the Lagrange multiplier trading between $D$ and $R$. RDO ultimately minimizes the R-D cost function $J$ defined as:

$$
J=(D+\lambda \times R)
$$

For video compression standards, such as High Efficiency Video Coding (HEVC) [2], source signal is processed by three operations known as prediction, transformation and quantization. $D$ measures the pixel-wise distance between source and decoded signals. $R$ is the amount of bits from quantized residues and syntax data coded with the entropic encoder.

In HEVC Intra coding [2], [3], frames are first equally divided into Coding Tree Units (CTUs). CTUs are square blocks of pixels processed in raster scan order. Each CTU is further recursively split, using a Quad-Tree, into multiple Coding Units (CUs) with possible sizes of $64 \times 64,32 \times 32$, $16 \times 16$ and $8 \times 8$. A CU of size $2 \mathrm{Nx} 2 \mathrm{~N}$ is composed of one Prediction Unit (PU) of the same size or four PUs of size $\mathrm{NxN}$ for the last depth of the CTU (with $N=4$ ). A prediction mode is set for each PU.
In this paper, we focus on Intra coding only and more precisely on the optimization of prediction mode decisions made at PU level. $P U_{i}$ denotes the $i_{t h} \mathrm{PU}$ to be encoded with respect to scanning order and $p_{i}$ denotes the coding parameters, i.e. intra prediction mode, of $P U_{i}$. With $N b$ the number of PUs to process, $R D O$ estimates:

$$
\left\{p_{k}^{*}\right\}_{k=0}^{N b-1}=\underset{\left\{p_{k}\right\}_{k=0}^{N b-1}}{\arg \min } \sum_{i=0}^{N b-1}\left(D_{\left.i\right|_{p_{i}}}+\lambda \times R_{\left.i\right|_{p_{i}}}\right)
$$

Since each PU is processed independently, (2) is optimal only if there is no dependency between PUs. Nevertheless, both predictive coding and Context Adaptive Binary Arithmetic Coding (CABAC) introduce dependencies between PUs.

Several studies aimed to take advantage of Inter-Block dependencies to achieve better coding efficiency [4]-[7]. In a previous work, we made explicit the dependencies related to predictive coding and CABAC [8]. Then, we proposed to achieve Joint-RDO (JRDO) on a pair of PUs. The model is called Dual-JRDO and consists in deciding the current predictor $p_{i}$ based on both its coding efficiency for current PU $\left(P U_{i}\right)$ and its impact on the following PU $\left(P U_{i+1}\right)$ coding efficiency, under a set of constraints.

The original model brings systematic bitrate savings (up to $1.12 \%$ ) for similar quality, but suffers from a significant computational complexity increase: encoding is 9 times slower. This paper provides efficient acceleration methods for Dual-JRDO, leading to a Fast Dual-JRDO model. The first proposed solution consists in enabling or disabling the model based on estimation of the spatial prediction complexity of the source, referred to as spatial activity in this paper. The two other proposed solutions consist in adaptively limiting the number of prediction modes competing in the joint analysis.

The remaining of the paper is organized as follows. A brief review of Dual-JRDO and its computational complexity are first given in Section II. Section III depicts the proposed acceleration solutions. Experimental results of the proposed Fast Dual-JRDO model are presented and discussed in Section IV. Finally, Section V concludes this paper.

\section{Dual-JRDO METHOD}

Let us consider two PUs $P U_{i}, P U_{j}$ with $i<j$ and $i, j \in$ $\{0, \ldots, N b-1\}$, where $N b$ is the number of PUs and $p_{i}$ is 
the prediction mode associated with $P U_{i}$. Two Inter-Block dependencies impact Intra coding. First, prediction error is dependent on the quantization error which affects reference samples. Second, $p_{j}$ signaling cost in the bitstream depends on $p_{i}$, and all CABAC contexts used to compute $R_{j}$ depend on probabilities update performed when encoding $P U_{i}$. By design, the optimal coding for $P U_{j}$ depends on $p_{i}$.

Therefore, we proposed in [8] to jointly optimize PUs subject to these dependencies, i.e. $j=i+1$. Dual-JRDO is summarized in Algorithm 1. Dual-JRDO is used when $P U_{i+1}$ is the spatial right neighbor of $P U_{i}$, otherwise classical-RDO is used to compute $p_{i}^{*}$.

$$
\begin{aligned}
& \text { Data: }\left\{P U_{i}\right\}_{i \overline{\bar{b}} 0}^{N b-1} \\
& \text { Result: }\left\{p_{i}^{*}\right\}_{i=0}^{N=0} \\
& \text { if isRight }\left(P U_{i+1}, P U_{i}\right) \text { then } \\
& \qquad p_{i}^{*}=\underset{p_{i}}{\arg \min }\left(J_{\left.i\right|_{p_{i}}}+\left.\underset{p_{i+1}}{\min } J_{i+1}\right|_{p_{i}, p_{i+1}}\right) \\
& \text { else } \\
& \text { end }
\end{aligned}
$$

\section{Algorithm 1: Dual-JRDO Algorithm}

During the estimation of $p_{i}^{*}, P U_{i}$ and $P U_{i+1}$ are assumed to be of the same size. An example of Dual-JRDO is shown in Figure 1, with $d$ the depth equivalent to PU size. Dotted lines delimit the optimization area, i.e. containing both $P U_{i}$ and $P U_{i+1}$; dark gray areas refer to blocks coded independently using classical-RDO, i.e. $P U_{i+1}$.

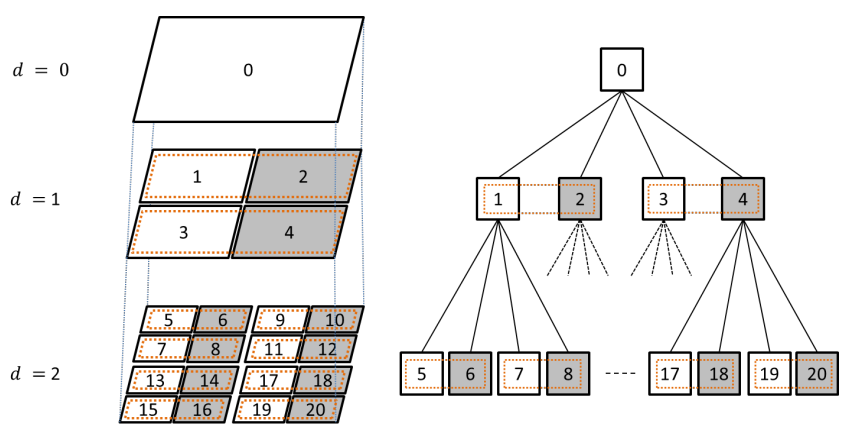

Fig. 1. Example of Dual-JRDO on a 3-level Quad-Tree

The next section presents the three proposed methods aiming to accelerate the Dual-JRDO coding scheme.

\section{ACCEleration Methods}

In this section, three methods are introduced in order to reduce the computational complexity $C p x$ of the Dual-JRDO. $C p x$ increase is directly related to $N b$, the number of analyzed PUs (of all size) in a frame during $R D O$. In the case of a single CTU of size $64 \times 64, N b=341$ : 1 PU 64x64, 4 PUs 32x32, 16 PUs $16 \times 16,64$ PUs $8 \times 8$ and 256 PUs $4 \times 4$. We note that $\mathrm{Nb}$ is composed of $93.84 \%$ of PUs with size of $4 \times 4$ or $8 \times 8$.
TABLE I

THE Dual-JRDO GAIN AGAINST classical-RDO FOR EACH DEPTH

\begin{tabular}{|c|c|c|c|c|c|c|}
\hline PU size & $64 \times 64$ & $32 \times 32$ & $16 \times 16$ & $8 \times 8$ & $4 \times 4$ & All \\
\hline Average & $0.00 \%$ & $0.00 \%$ & $-0.06 \%$ & $-0.11 \%$ & $-0.46 \%$ & $\mathbf{- 0 . 6 3 \%}$ \\
\hline Maximum & $0.00 \%$ & $-0.08 \%$ & $-0.28 \%$ & $-0.19 \%$ & $-1.02 \%$ & $\mathbf{- 1 . 1 2 \%}$ \\
\hline Minimum & $0.00 \%$ & $+0.23 \%$ & $+0.04 \%$ & $+0.05 \%$ & $-0.01 \%$ & $\mathbf{- 0 . 1 9 \%}$ \\
\hline
\end{tabular}

Additional experiments, illustrated in Table I, assess the BDrate gain of Dual-JRDO brought by each PU size (or specific depth). Test conditions are the same as described in Section IV. These results show that the R-D gains introduced by Dual$J R D O$ are mostly brought by coding efficiency improvement of $4 \times 4$ and $8 \times 8$ PUs. In the following, one focuses on accelerating Dual-JRDO applied to $4 \times 4$ and $8 \times 8$ PUs because of their high complexity. In practice, $64 \times 64 \mathrm{PU}$ size rarely appear to be optimal, which explains that no gains are observed here.

\section{A. Adapting to Spatial Activity}

Quad-Tree is responsible for the large $C p x$ endured by HEVC [9]. Since Intra coding favors large PU size for smooth areas and small PU size for textured areas, many fast algorithms [10]-[12] estimate the spatial activity of the source to adaptively skip $R D O$ for some PUs.

Thus, we propose to rely on a spatial activity measure, quite similar to the one defined in [10], for adaptive use of Dual$J R D O$. In the case of high activity, classical-RDO is supposed to be sub-optimal and thus we activate Dual-JRDO.

As a good trade-off between metric computational overhead and estimator accuracy, the spatial activity is computed over $16 \times 16$ pixel area. Furthermore, in order to be more robust to random noise, the computation is done on a $16 \times 16$ block down-sampled to $4 \times 4$. Note that sub-blocks $(16 \times 8$ or $8 \times 4)$ take the same spatial activity value which is computed from the corresponding upper 16x16 size bloc, as described below:

1) Down-sample all 16x16 blocks into $4 \times 4$ blocks, then compute spatial activity $g_{i}$ as defined in (5)

2) Each PU of $4 \times 4$ and $8 \times 8$ size is assigned with $g_{i}$ value of the corresponding $16 \times 16 \mathrm{PU}$

3) If $g_{i} \geq T h, P U_{i}$ is processed with Dual-JRDO

$$
g_{i}=\frac{1}{16} \sum_{x=0}^{3} \sum_{y=0}^{3} \min \left\{\begin{array}{l}
\left|I_{i}(x, y)-I_{i}(x-1, y)\right| \\
\left|I_{i}(x, y)-I_{i}(x, y-1)\right|
\end{array}\right.
$$

with $I_{i}(x, y)$ the pixel luminance value at relative position $(x, y)$ of down-sampled $16 \times 16 P U_{i}$. In order to exclude neighboring PUs energy, we set $I_{i}(-1, y)=I_{i}(x,-1)=0$.

$T h$ is a predefined threshold computed off-line with a supervised learning using logistic regression [13]. Dual-JRDO estimation encloses classical-RDO estimation by design, hence we can a posteriori observe if Dual-JRDO was of interest.

Supervised learning is used to estimate the optimal threshold for five $Q P$ values: $22,27,32,37,42$. The relationship between $Q P$ and $T h$ is then obtained through logistic least square method applied on the previously obtained $(Q P, T h)$ couples as: 


$$
T h(Q P)=\alpha \times e^{\beta \times Q P}
$$

with $(\alpha, \beta)$ values equal to $(0.0963,0.107)$ in our case.

During threshold learning, we observe that the classification is more efficient for high $Q P$ values. One possible explanation is that for low rates, $D$ tends to be equal to the prediction error. Consequently, since the spatial activity is a coarse estimation of the difficulty to predict a couple of PUs, it becomes at low rates a better predictor of the need to activate Dual-JRDO.

\section{B. Short Listing on $P U_{i+1}$}

In the HEVC reference Model HM16.12 [14] used in our experiments, classical-RDO for Intra coding is only applied on a shortlist of modes created by Rough Mode Decision ( $R M D)$ algorithm [3]. RMD consists in short-listing prediction modes with lowest residual Sum of Absolute Transform Differences (SATD) value and syntax cost. Only this short-list is then estimated through RDO. In addition to the three Most Probable Modes MPMs (which are always evaluated), the minimal number of modes to be considered in $R D O$ is respectively set to 8 for $4 \times 4$ and $8 \times 8$ PUs, and 3 for larger PU sizes.

The set of coding modes to consider (i.e. the possible values of $p_{i}$ ) is denoted $M_{i}$. In Dual-JRDO, $M_{i+1}$ set consists of 35 intra prediction modes defined in HEVC. Since $p_{i+1}^{*}$ is selected by the $R M D$ process in the HM software, it seems relevant to also construct the $M_{i+1}$ list based on the $R M D$ optimization. Residual, syntax mode cost and $M P M$ s being all dependent of $p_{i}$, we denote as $M_{i+1}\left(p_{i}\right)$ the set of modes to be considered for $P U_{i+1}$ while optimizing $P U_{i}$. Therefore, (3) becomes (7) as:

$$
p_{i}^{*}=\underset{p_{i} \in M_{i}}{\arg \min }\left(J_{\left.i\right|_{p_{i}}}+\min _{p_{i+1} \in M_{i+1}\left(p_{i}\right)} J_{i+\left.1\right|_{p_{i}, p_{i+1}}}\right)
$$

Note that $R M D$ is inherited from the $R D O$ implementation in the HM16.12. However, any conceivable short-listing approach efficient for HEVC intra coding with classical-RDO model, could be also beneficial for the Dual-JRDO model.

\section{Prediction Modes Clustering based on Residual Analysis}

Two sources of dependencies between PUs have been exhibited in Section II: Distortion and CABAC. The CABAC dependency is considered negligible in Dual-JRDO coding scheme since both $P U_{i}$ and $P U_{i+1}$ are subject to very similar contexts. We assume that $P U_{i+1}$ is then affected by a simple dependency: the distortion made on $P U_{i}$.

We assume that two prediction modes which result into identical residual signal also result in identical reconstructed signal. This assertion is true if no divergent process impacts the coding of residual. It implies identical transformation and quantization steps for HEVC Intra coding.

Mode Dependent Coding Scan (MDCS) [15] used in Intra HEVC does not fulfill the requirement of no mode-dependent process on residuals. However, we ignore the minor difference of process attributed to $M D C S$ since it has very little impact on the proposed solution efficiency.
By considering only distortion dependency and the correlation between prediction residual and reconstructed data, we suppose that two modes of $P U_{i}$ resulting in the same residual data share the exact same impact on $P U_{i+1}$. Consequently, we define as a cluster a set of prediction modes which result into identical residual signal. Let $p_{i} 1$ and $p_{i} 2$, two coding parameters of $P U_{i}$ which result into the same prediction residual. Under the previous statement, equality (8) holds.

$$
\min _{p_{i+1}}\left(J_{i+\left.1\right|_{p_{i} 1, p_{i+1}}}\right)=\min _{p_{i+1}}\left(J_{i+\left.1\right|_{p_{i} 2, p_{i+1}}}\right)
$$

And from (8) we can write (9) and (10).

$$
\begin{gathered}
p_{i}^{*}=\underset{p_{i}}{\arg \min }\left(J_{\left.i\right|_{p_{i}}}+J_{i+\left.1\right|_{p_{i+1}^{\prime}}}\right) \\
p_{i+1}^{\prime}=\underset{p_{i+1}}{\arg \min }\left(J_{i+\left.1\right|_{p_{i}, p_{i+1}}}\right)
\end{gathered}
$$

which is correct with all possible $p_{i}$ remaining in the same cluster. $p_{i+1}^{\prime}$ is defined as the optimal $P U_{i+1}$ coding mode for all $p_{i}$ in the cluster. Consequently, $p_{i+1}^{\prime}$ estimation is common for all $p_{i}$ in the same cluster.

This method is summarized in three steps:

1) Construct the different clusters by analyzing mode residuals while $R M D$ is applied to $P U_{i}$

2) If $p_{i}$ is the first of its cluster, optimize $p_{i+1}$ among all possible modes

3) Otherwise, optimize $p_{i+1}$ among previous $p_{i+1}^{*}$ of the same cluster and new MPMs modes

Many bits are saved if the optimal mode belongs to MPMs. Consequently, the third step ensures that MPMs of $p_{i+1}$ are always tested if they differ from the $M P M s$ previously considered within the cluster, i.e. if different from $p_{i}$. This technique is an effective shortcut as long as the number of final clusters is low, which is often verified for small CU sizes.

\section{EXPERIMENTS AND RESUlTS}

Acceleration methods of Section III have been implemented in HM16.12 with the Dual-JRDO algorithm. Results are presented with five configurations $\left\{C_{k}\right\}_{k=0}^{4}$ summarized in Table II. The reference is HM16.12 with classical-RDO. Impacts of each solution on both $C p x$ and R-D efficiency are individually evaluated. For comparison purpose, we include results of Dual-JRDO in HM16.12 without acceleration $\left(C_{0}\right)$.

TABLE II

CONFIGURATIONS

\begin{tabular}{|l|c|c|c|c|c|}
\hline Configurations & $C_{0}$ & $C_{1}$ & $C_{2}$ & $C_{3}$ & $C_{4}$ \\
\hline Spatial Activity Adaptation & & $\mathrm{x}$ & & & $\mathrm{x}$ \\
\hline Short-List $M_{i+1}$ & & & $\mathrm{x}$ & & $\mathrm{x}$ \\
\hline Residual Based Clustering & & & & $\mathrm{x}$ & $\mathrm{x}$ \\
\hline
\end{tabular}

Test conditions follow the recommendations of the Joint Collaborative Team on Video Coding (JCT-VC) [16] in All-Intra configuration. Coding efficiency is measured using Bjøntegaard BD-BR [17] with Peak Signal to Noise Ratio 
(PSNR). Since, BD-BR is the difference of areas under two R-D functions, we choose to add a fifth R-D point at $Q P=42$ in order to cover a larger bitrate range with the same metric. We use the configuration files provided with HM16.12.

For this experiment, YUV BD-BR results of each configuration against classical-RDO are presented in Table III. Cpx savings over initial Dual-JRDO $\left(C_{0}\right)$ are presented in Table IV. $C p x$ saving is estimated according to (11), with encoding times Time ref and Time current of HM16.12 with Dual-JRDO without modification and Dual-JRDO with the proposed optimizations, respectively.

$$
C p x(\%)=\frac{\text { Time }_{\text {current }}-\text { Time }_{\text {ref }}}{\text { Time }_{\text {ref }}} * 100
$$

We observe systematic bitrate savings against classical$R D O$ for all considered coding configurations. However, the more aggressive is the algorithm, in terms of $C p x$ reduction, the less efficient Dual-JRDO is.

TABLE III

Dual-JRDO CODING EFFICIENCY OVER classical-RDO IN HM16.12.

\begin{tabular}{|c|c|c|c|c|c|}
\hline Classes & $C_{0}$ & $C_{1}$ & $C_{2}$ & $C_{3}$ & $C_{4}$ \\
\hline \hline Class B & $-0.45 \%$ & $-0.42 \%$ & $-0.38 \%$ & $-0.46 \%$ & $-0.35 \%$ \\
\hline Class C & $-0.61 \%$ & $-0.54 \%$ & $-0.47 \%$ & $-0.61 \%$ & $-0.42 \%$ \\
\hline Class D & $-0.63 \%$ & $-0.59 \%$ & $-0.46 \%$ & $-0.64 \%$ & $-0.44 \%$ \\
\hline Class E & $-0.64 \%$ & $-0.58 \%$ & $-0.52 \%$ & $-0.64 \%$ & $-0.47 \%$ \\
\hline Class F & $-0.87 \%$ & $-0.76 \%$ & $-0.67 \%$ & $-0.88 \%$ & $-0.60 \%$ \\
\hline \hline Average & $\mathbf{- 0 . 6 3 \%}$ & $\mathbf{- 0 . 5 7 \%}$ & $\mathbf{- 0 . 4 9 \%}$ & $\mathbf{- 0 . 6 3 \%}$ & $\mathbf{- 0 . 4 5 \%}$ \\
\hline Maximum & $\mathbf{- 1 . 1 2 \%}$ & $\mathbf{- 1 . 0 1 \%}$ & $\mathbf{- 0 . 8 7 \%}$ & $\mathbf{- 1 . 1 1 \%}$ & $\mathbf{- 0 . 8 2 \%}$ \\
\hline Minimum & $\mathbf{- 0 . 1 9 \%}$ & $\mathbf{- 0 . 2 1 \%}$ & $\mathbf{- 0 . 2 0 \%}$ & $\mathbf{- 0 . 2 0 \%}$ & $\mathbf{- 0 . 2 0 \%}$ \\
\hline
\end{tabular}

TABLE IV

Dual-JRDO COMPLEXITY OVER $C_{0}$ CONFIGURATION.

\begin{tabular}{|c|c|c|c|c|}
\hline Classes & $C_{1}$ & $C_{2}$ & $C_{3}$ & $C_{4}$ \\
\hline \hline Class B & $-49.94 \%$ & $-75.21 \%$ & $-13.01 \%$ & $-83.80 \%$ \\
\hline Class C & $-35.08 \%$ & $-76.13 \%$ & $-14.13 \%$ & $-81.12 \%$ \\
\hline Class D & $-20.42 \%$ & $-72.25 \%$ & $-0.42 \%$ & $-77.61 \%$ \\
\hline Class E & $-56.59 \%$ & $-74.26 \%$ & $-23.30 \%$ & $-85.28 \%$ \\
\hline Class F & $-46.60 \%$ & $-74.35 \%$ & $-20.16 \%$ & $-83.90 \%$ \\
\hline \hline Average & $\mathbf{- 4 1 . 7 9 \%}$ & $\mathbf{- 7 4 . 6 2 \%}$ & $\mathbf{- 1 3 . 9 7 \%}$ & $\mathbf{- 8 2 . 4 4 \%}$ \\
\hline Maximum & $\mathbf{- 2 5 . 8 9 \%}$ & $\mathbf{- 7 5 . 8 3 \%}$ & $\mathbf{- 1 4 . 8 4 \%}$ & $\mathbf{- 7 9 . 6 3 \%}$ \\
\hline Minimum & $\mathbf{- 7 1 . 0 6 \%}$ & $\mathbf{- 7 2 . 7 8 \%}$ & $\mathbf{- 3 3 . 2 4 \%}$ & $\mathbf{- 8 9 . 5 4 \%}$ \\
\hline
\end{tabular}

Adaptive activation of the model based on spatial activity corresponds to configuration $C_{1}$. In average $C p x$ reduction is about $41.79 \%$ for $0.06 \%$ BD-BR loss. The slight observed loss for $C_{1}$ can be explained by the off-line learning to approximate the threshold $T h$ used in the decision.

Configuration $C_{2}$ uses $R M D$ during the $p_{i+1}^{\prime}$ estimation. It is one of the most efficient in terms of $C p x$ reduction. We observe average $C p x$ decrease of $74.62 \%$ against $C_{0}$ configuration, at the cost of an average BD-BR increase of $0.14 \%$. The results of this solution imply that any short-listing approach efficient into classical-RDO can be easily transposed into Dual-JRDO framework.

Configuration $C_{3}$ uses prediction mode clustering based on residual analysis. BD-BR gains are better preserves by suppressing redundant coding process without any approximation.
TABLE V

FAST Dual-JRDO CODING EFFICIENCY AND COMPUTATIONAL COMPLEXITY OVER classical-RDO IN HM16.12.

\begin{tabular}{|c|c|c|}
\hline Classes & $\Delta$ Bit-rate $(\%)$ & Encoding Time $(\%)$ \\
\hline Class B & $-0.35 \%$ & $133 \%$ \\
\hline Class C & $-0.42 \%$ & $156 \%$ \\
\hline Class D & $-0.44 \%$ & $160 \%$ \\
\hline Class E & $-0.47 \%$ & $116 \%$ \\
\hline Class F & $-0.60 \%$ & $124 \%$ \\
\hline \hline Average & $\mathbf{- 0 . 4 5 \%}$ & $\mathbf{1 3 8 \%}$ \\
\hline Maximum & $\mathbf{- 0 . 8 2 \%}$ & $\mathbf{1 7 8 \%}$ \\
\hline Minimum & $\mathbf{- 0 . 2 0 \%}$ & $\mathbf{7 4 \%}$ \\
\hline
\end{tabular}

Experimental observations show that cases of identical residual for different predictors occur rarely in textured content. The computational cost of comparing all residuals is also a nonnegligible overhead. These two facts explain why $C p x$ is not significantly decreased (-13.97\% in average).

Configuration $C_{4}$ represents the combination of the three solutions from Section III. For each PU, the algorithm equivalent to $C_{1}$ decides whether Dual-JRDO is to be used or not. Next, algorithm corresponding to $C_{3}$ configuration builds the mode clusters based on $R M D$ process. Finally, for the first tested mode of each cluster, $R M D$ is enabled while analyzing $P U_{i+1}$. For any new mode that belongs to the same cluster, the solution described by Section III-C is applied. The final Fast Dual-JRDO combination limits $C p x$ increase to $138 \%$ against classical-RDO, with an average BD-BR of $-0.45 \%$ and up to $-0.82 \%$, as shown in Table V.

\section{CONClusion}

In this paper, we have proposed three acceleration methods to benefit from Inter-Block dependencies and improve HEVC Intra coding efficiency without tremendous computational cost.

Considering the Intra prediction mode as the coding parameter to optimize, we observe a correlation between the source spatial activity and the Dual-JRDO effectiveness. Thus, a robust spatial activity metric, is first designed to efficiently activate or not the Dual-JRDO model for each PU. Besides, $R M D$ short-listing algorithm is successfully integrated in the $p_{i+1}^{\prime}$ estimation. Finally, we introduced a prediction mode clustering approach that suppresses redundant computations for modes resulting in identical residue. This last acceleration method is based on the fact that similar residue on $P U_{i}$ should lead to the same impact on $P U_{i+1}$ optimal coding.

The combination of the three proposed acceleration methods results in a Fast Dual-JRDO that improved HEVC coding efficiency by $-0.45 \%$ BD-BR for a computational complexity of $138 \%$ in comparison to the HM16.12 reference model.

Our future work will focus on dependencies related to Interframes prediction, more subject to distortion propagation onto temporal axes, which could lead to higher gains for JRDO approaches. We also note that in this paper, Dual-JRDO focus on optimizing intra prediction modes couples. However, one can easily extend the model to other coding parameters, such as $Q P$ for $\mathrm{CU}$ couples. 


\section{REFERENCES}

[1] T. Wiegand and B. Girod, "Lagrange multiplier selection in hybrid video coder control," in International Conference on Image Processing (ICIP). Thessaloniki: IEEE, Oct. 2001, pp. 542-545.

[2] G.-J. Sullivan, J.-R. Ohm, W.-J. Han, and T. Wiegand, "Overview of the High Efficiency Video Coding (HEVC) Standard," IEEE Transactions on Circuits and Systems for Video Technology (TCSVT), vol. 22, no. 12, pp. 1649-1668, Dec. 2012.

[3] J. Lainema, F. Bossen, W.-J. Han, J. Min, and K. Ugur, "Intra Coding of the HEVC Standard," IEEE Transactions on Circuits and Systems for Video Technology, vol. 22, no. 12, pp. 1792-1801, Dec. 2012.

[4] C. Pang, O. C. Au, F. Zou, J. Dai, and R. Cha, "Optimal distortion redistribution in block-based image coding using successive convex optimization," in International Conference on Multimedia and Expo (ICME). IEEE, 2011, pp. 1-5.

[5] C. Pang, O. C. Au, F. Zou, X. Zhang, W. Hu, and P. Wan, "Optimal dependent bit allocation for AVS intra-frame coding via successive convex approximation," in International Conference on Image Processing (ICIP). IEEE, 2013.

[6] L. Sun, O. C. Au, W. Dai, R. Zou, and S. Li, "Modified distortion redistribution problem for High Efficiency Video Coding (HEVC)," in International Workshop on Multimedia Signal Processing (MMSP). IEEE, 2012, pp. 278-282.

[7] W. U. Qingbo, J. Xiong, L. U. O. Bing, C. Huang, and X. U. Linfeng, "A Novel Joint Rate Distortion Optimization Scheme for Intra Prediction Coding in H. 264/AVC," IEICE Transactions on Information and Systems, vol. 97, no. 4, pp. 989-992, 2014.

[8] M. Bichon, J. Le Tanou, M. Ropert, W. Hamidouche, L. Morin, and L. Zhang, "Inter-Block Dependencies Consideration for Intra Coding in H.264/AVC and HEVC standards," in IEEE International Conference on Acoustics, Speech and Signal Processing (ICASSP), 2017.

[9] A. Mercat, A. Arrestier, W. Hamidouche, M. Pelcat, and D. Menard, "Energy Reduction Opportunities in an HEVC Real-Time Encoder," in IEEE International Conference on Acoustics, Speech and Signal Processing (ICASSP), 2017.

[10] G. Tian and S. Goto, "Content adaptive prediction unit size decision algorithm for HEVC intra coding," in Picture Coding Symposium (PCS), May 2012, pp. 405-408.

[11] Y. Shi, O. Au, X. Zhang, H. Zhang, R. Ma, and L. Jia, "Content adaptive prediction unit size decision algorithm for HEVC intra coding," in IEEE International Symposium on Circuits and Systems (ISCAS), 2013, pp. 225-228.

[12] Y. Liu, Z. Chen, J. Fang, and P. Chang, "SVM-Based Fast Intra CU Depth Decision for HEVC," in IEEE Data Compression Conference (DCC), Apr. 2015, p. 458.

[13] S. le Cessie and J. van Houwelingen, "Ridge estimators in logistic regression," Applied Statistics, vol. 41, no. 1, pp. 191-201, 1992.

[14] K. McCann, C. Rosewarne, B. Bross, M. Naccari, K. Sharman, and G. Sullivan, "JCTVC-R1002: High Efficiency Video Coding (HEVC) Test Model 16 (HM 16) Encoder Description," Jul. 2014.

[15] J. Sole, R. Joshi, N. Nguyen, T. Ji, M. Karczewicz, G. Clare, F. Henry, and A. Duenas, "Transform Coefficient Coding in HEVC," IEEE Transactions on Circuits and Systems for Video Technology, vol. 22, no. 12, pp. 1765-1777, Dec. 2012.

[16] F. Bossen, "Common test conditions and software reference configurations," Tech. Rep. JCTVC-L1100, Joint Collaborative Team on Video Coding (JCT-VC), Jan. 2013.

[17] G. Bjontegaard, "Calculation of average PSNR differences between RDcurves," in ITU-T VCEG, Texas, USA, Proposal VCEG-M33, Austin, Texas, Apr. 2001. 